\title{
Synchronous Tuberculosis and Breast Cancer Metastasis in Axillary Lymph Nodes: A Case Report
}

\author{
Ramanjis Tallari ${ }^{1}$, Veenita Yogi ${ }^{2}$, O.P. Singh ${ }^{3}$ \\ ${ }^{1}$ Junior Resident IIIrd year, Department of Radiation Oncology, Gandhi Medical College, Bhopal,(M.P.) India \\ ${ }^{2}$ Assistant Professor, Department of Radiation Oncology, Gandhi Medical College, Bhopal,(M.P.) India \\ ${ }^{3}$ Professor and Head, Department of Radiation Oncology, Gandhi Medical College, Bhopal,(M.P.) India
}

\begin{abstract}
Synchronous presence of tuberculosis and breast cancer metastasis in axillary lymph nodes is rare. Tuberculosis ranks as the second leading cause of death from an infectious disease worldwide, after the human immunodeficiency virus(HIV). We present here a case report of infiltrating ductal carcinoma (IDC) of the left breast with axillary lymphadenitis harbouring both tuberculosis and metastatic cancer cells from breast cancer at the same time. Simultaneous presence of both diseases make treatment difficult as tuberculosis require long course of anti-tuberculosis drugs and reduces patient compliance. In this case, breast cancer was treated by neoadjuvant chemotherapy, surgery and adjuvant chemoradiotherapy and axillary lymph nodal tuberculosis was treated by antituberculosis drugs.
\end{abstract}

Keywords: Axillary lymphadenitis, Breast cancer, Tuberculosis, chemotherapy, anti-tuberculosis drugs.

\section{Introduction}

There are two forms of tuberculosis, pulmonary and extrapulmonary.

Extrapulmonary Tuberculosis (TB) constitutes about 15 to 20 per cent of all cases of tuberculosis with lymph nodes being the most common site of involvement [1].

The simultaneous occurrence of tuberculosis (TB) and cancer in breast is rare, and the simultaneous occurrence of tuberculosis of axillary lymph nodes and cancer metastasis in axillary lymph nodes from breast cancer is even rarer.

Tuberculosis ranks as the second leading cause of death from an infectious disease worldwide after the human immunodeficiency virus(HIV)[2], and is responsible for high rate of mortality and morbidity mainly in the developing countries. All the organs of the human body are susceptible to it except hairs and nails.

Co-existing TB with cancer has been previously reported in most organs, especially with lung cancer. Co-existing carcinoma breast with TB axillary lymph node may lead to a false overstaging of the disease. We report a case of infiltrating ductal carcinoma of the left breast, metastatic to ipsilateral axillary lymph nodes harbouring tuberculous lymphadenitis without primary breast or pulmonary tuberculosis. The simultaneous occurrence of tuberculosis and carcinoma can create a dilemma in the diagnosis and treatment.

\section{Case Report}

A 53 year old female patient presented to our outpatient department with history of painless, mobile,gradually increasing lump in the left breast since 1 year, gradually increasing lump in the left axilla since 8 months. Personal history was not significant, menopausal since last 1 year.

There was contact history of TB within her family, as two of her sons were victims of pulmonary TB. On examination there was a firm lump in the left breast, in the upper inner and outer quadrant measuring $3 * 3 \mathrm{~cm}$. Multiple firm left axillary lymph nodes also palpable, largest measuring $4 * 3 \mathrm{~cm}$ in size, contralateral breast and axilla were normal. There was a midline swelling over the anterior aspect of the neck region most probably a long standing goiter.

Routine blood and thyroid function tests were within normal limits, montoux test reported positive, chest x-ray was reported normal, fine needle aspiration cytology(FNAC) thyroid swelling revealed cystic lesion of thyroid.

Breast lump FNAC reported as ductal carcinoma.

Patient was planned for neo-adjuvant chemotherapy followed by surgery followed by adjuvant chemo-radiotherapy. She underwent modified radical mastectomy (MRM) after 3 cycles of paclitaxel based neo-adjuvant chemotherapy.

Post MRM histopathological report revealed, infiltrating ductal carcinoma(IDC) grade III left breast with axillary lymph node metastasis(modified bloom Richardson score$3+3+3=9$ ) along with axillary lymph node tuberculosis(Figure1). 


\section{International Journal of Science and Research (IJSR) \\ ISSN (Online): 2319-7064}

Index Copernicus Value (2013): 6.14 | Impact Factor (2014): 5.611

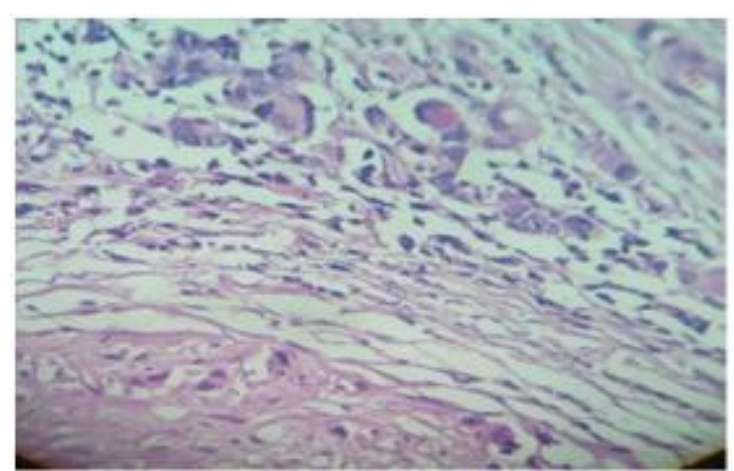

Figure 1: H\&E staining,high power view of axillary lymph node showing both infitrating ductal cancer cells with TB granuloma.

4/27 axillary lymph nodes showing both cancer metastasis and TB granuloma while majority of remaining nodes showing necrotizing granulomas with langhan's type giant cells consistent with tuberculosis(Figure2).

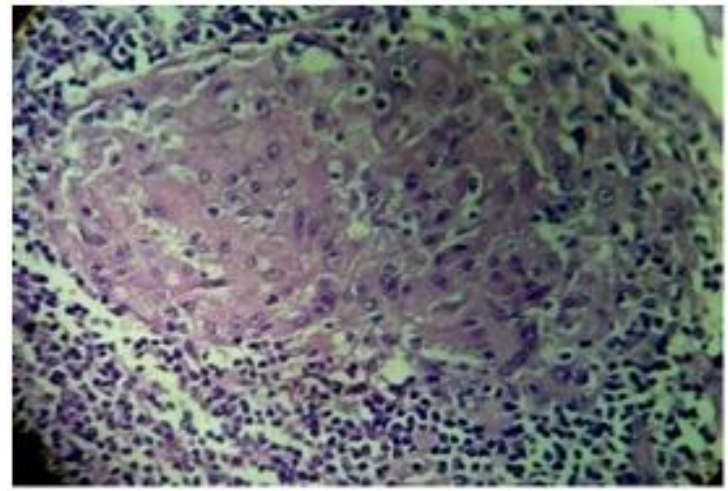

Figure 2: H\&E staining high power view axillary lymph node showing of TB granuloma

and (Figure3) showing IDC breast.

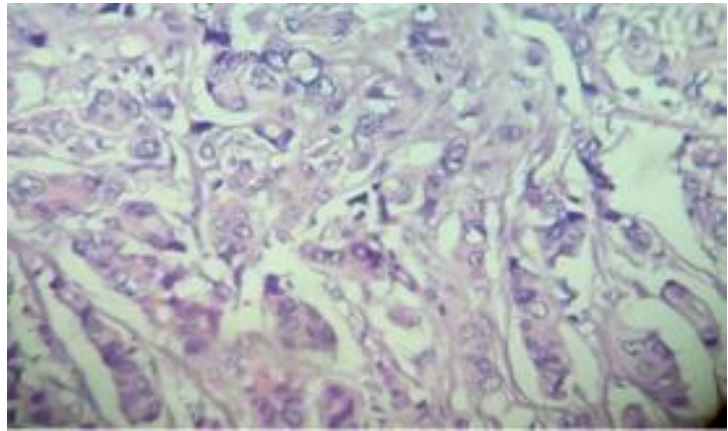

Figure 3: H\&E staining high power view of breast mass showing infitrating ductal cancer cells.

Patient was started on anti-TB drugs through DOTS centre near her residence and recently she has completed her DOTS course with good response.

\section{Discussion}

Robert Koch in 1882 discovered TB bacilli and till date it's more than 130 years now, we are battling with tuberculosis. Tuberculosis (TB) remains one of the world's deadliest communicable diseases. In 2013, an estimated 9.0 million people developed TB and 1.5 million died from the disease[2]

The synchronous occurrence of tuberculosis and cancer existing in the same patient was first described by Warthin in 1899[3].

Kaplan et al. reviewed 58,245 patients with cancer and identified 201 cases of co-existing tuberculosis [4].

Tuberculous lymphadenitis is the commonest form of extrapulmonary tuberculosis and cervical lymph nodes is the most common site of involvement. Primary axillary lymph node involvement in adults is uncommon and in this case it could be either a retrograde spread from the mediastinal nodes, or haematogenous spread from a subclinical focus and could be activation process of quiescent tubercular infection of axillary lymph node due to immuno-suppression in cancer patients.

While both breast cancer and tuberculosis (TB) are common in developing countries, their co-existence in the axillary nodes is rare and few reported in literature[5-10]. Some of these are Pandey $\mathrm{M}$ et al[7], reported infiltrating ductal carcinoma metastatic to axillary lymph nodes containing tuberculous foci, Das et al[9], reported a case of colloid carcinoma of the breast metastatic to axillary lymph nodes, Pandey $\mathrm{P}$ et al[10], reported infiltrating ductal carcinoma breast, metastatic to ipsilateral axillary lymph nodes harbouring primary tuberculous lymphadenitis.

\section{Conclusion}

Breast cancer presenting with axillary lymph nodes should be evaluated for presence of tuberculosis too, which will help in correct staging of the disease and treatment.

Axillary lymph node enlargement in breast cancer patient is not always caused by metastatic tumor of the breast even in the ipsilateral axillary nodes. Accurate diagnosis will help in down staging carcinoma of the breast and also in identifying curable disease.

\section{References}

[1] Sharma SK, Mohan A. Extrapulmonary tuberculosis.A review article. Indian J Med Res 2004;120: 316-53.

[2] WHO Global tuberculosis report 2014 chapter one.

[3] Warthin AS. The coexistence of tuberculosis and carcinoma of the mammary gland. Am J Med Sci 1899; 118: 25.

[4] Kaplan MH, Armstrong D, Rosen P. Tuberculosis complicating neoplastic disease: a review of 201 cases. Cancer 1974; 33: 850-8.

[5] Robinson AJ, Horne CA, Weaver A. Coexistence of axillary tuberculous lymphadenitis with lymph node metastases from a breast carcinoma. Clin Oncol (R Coll Radiol) 2001; 13: 144.

[6] Akbulut S, Sogutcu N,Yagmur Y. Coexistence of breast cancer and tuberculosis in axillary lymph nodes: a case report and literature review. Breast Cancer Res Treat. 2011 Dec;130(3):1037-42. doi: 10.1007/s10549-0111634-8. Epub 2011 Sep 22. Review. 


\section{International Journal of Science and Research (IJSR) \\ ISSN (Online): 2319-7064}

Index Copernicus Value (2013): 6.14 | Impact Factor (2014): 5.611

[7] Pandey M, Abraham EK, K C, Rajan B. Tuberculosis and metastatic carcinoma coexistence in axillary lymph node: a case report. World J Surg Oncol 2003; 1: 3 .

[8] Miller RE, Solomen PF, West JP. The coexistence of carcinoma and tuberculosis of the breast and axillary lymph nodes. Am J Surg 1971; 121: 338-40.

[9] Das DK, Mohil RS, Kashyap V, Khan IV, Mandal AK, Gulati SM. Colloid carcinoma of the breast with concomitant metastasis and a tubercular lesion in the axillary lymph nodes: a case report. Acta Cytol 1992; 36: 399-403.

[10] Pandey P, Dixit A, Tanwar A, Mahajan NC. Infiltrating ductal carcinoma breast, metastatic to ipsilateral axillary lymph nodes harbouring primary tuberculous lymphadenitis: a case report. Pan Afr Med J.2014Jun19;18:167.doi:10.11604/pamj.2014.18.167.14 94. e collection 2014 . 